\title{
Efficacy and safety of PCSK9 inhibition in cardiovascular disease: A meta-analysis of 45 randomized controlled trials
}

\author{
Qiang Geng, Xuan Li, Qingjiao Sun, Zhengzhong Wang
}

Department of Cardiology, Qingdao Municipal Hospital, Qingdao, Shandong, China

\begin{abstract}
Background: Safety concerns about proprotein convertase subtilisin/kexin type 9 (PCSK9) inhibitors make physicians reluctant to prescribe agents for patients. The present aim was to assess the efficacy and safety of alirocumab, evolocumab and bococizumab in patients with atherosclerotic cardiovascular disease (ASCVD).

Methods: Medline, the Cochrane Library and Clinicaltrials.gov were searched for 45 randomized controlled trials, involving 97,297 patients.

Results: Compared with the control group, PCSK9 inhibitors could significantly reduce low-density lipoprotein cholesterol, total cholesterol, triglycerides and increase high-density lipoprotein cholesterol. Alirocumab was associated with lower incidence of unstable angina $(p<0.05)$ and myocardial infarction $(p<0.05)$, compared with the control group. Alirocumab (odds ratio [OR] 0.76, 95\% confidence interval [CI] 0.60-0.97, $p<0.05)$, evolocumab (OR 0.79, 95\% CI 0.66-0.95, $p<0.05)$ and bococizumab (OR 0.60, 95\% CI 0.42-0.84, $p<0.05)$ were associated with lower incidence of stroke, compared with control group. The incidence of injection-site reactions was significantly higher in alirocumab $(O R$ 1.68, 95\% CI 1.45-1.93, $p<0.05)$, evolocumab (OR 1.64, 95\% CI 1.41-1.91, $p<0.05)$ and bococizumab (OR 8.03, 95\% CI 6.85-9.41, $p<0.05$ ) group than in the control group.

Conclusions: Alirocumab and evolocumab could ameliorate lipid profile and reduce the risk of cardiac disorders and stroke with satisfactory safety and tolerability. However, injection-site reactions should be paid attention to. (Cardiol J 2022; 29, 4: 574-581)

Key words: proprotein convertase subtilisin/kexin type 9, efficacy, safety, meta-analysis
\end{abstract}

\section{Introduction}

Statins were recommended as a first-line therapy for cardiovascular disease (CVD) and substantially decreased the risk for CVD events. But, a high proportion of patients did not achieve optimal levels of low-density lipoprotein cholesterol (LDL-C) or may have had high residual CVD risk despite high-intensity statin therapy. An optional approach is to choose other LDL-C lowering agents for these patients on the basis of statins.
Proprotein convertase subtilisin/kexin type 9 (PCSK9) promotes the degradation of low-density lipoprotein receptors (LDLR) at the surface of hepatocytes by binding to LDLR in lysosomes/ /endosomes. PCSK9 inhibitors have emerged as an effective strategy to reduce LDL-C and other lipid parameters. Alirocumab and evolocumab appeared to reduce nonfatal major adverse cardiac event (MACE) and be well tolerated [1]. But, further development of bococizumab was discontinued because of no significant reduction in cardiovas-

Address for correspondence: Dr. Zhengzhong Wang, Department of Cardiology, Qingdao Municipal Hospital, Qingdao, Shandong 266011, China, tel: +860532-82789351, e-mail: geng202004@163.com

Received: 23.03.2021 Accepted: 22.08.2021 Early publication date: 23.09.2021

This article is available in open access under Creative Common Attribution-Non-Commercial-No Derivatives 4.0 International (CC BY-NC-ND 4.0) license, allowing to download articles and share them with others as long as they credit the authors and the publisher, but without permission to change them in any way or use them commercially. 
cular events and high incidence of injection-site reactions with bococizumab $[2,3]$. Physicians were still worried about the efficacy and safety of PCSK9 inhibitors and reluctant to prescribe for atherosclerotic cardiovascular disease (ASCVD) patients. With the increase in evidence in recent years, this meta-analysis was therefore performed to evaluate the efficacy and safety of PCSK9 inhibitors (alirocumab, evolocumab, bococizumab) currently available in clinical practice.

\section{Methods}

\section{Search strategy}

This meta-analysis was performed in accordance with the Preferred Reporting of Systematic Reviews and Meta-Analysis (PRISMA) statement [4]. Pubmed, the Cochrane Library database, Clinicaltrials.gov from March 2012 to March 2021 were searched using the following search items: "evolocumab", "AMG 145", "alirocumab", “SAR236553”, "REGN727”, "bococizumab” and “RN316/PF-04950615”. The search pattern is shown in Supplementary Table S2.

\section{Study selection}

Two independent investigators screened article titles and full-text according to the inclusion criteria. Discrepancies were resolved by discussion and consensus. Risk of bias of was assessed by using the Cochrane Risk of Bias tool [5].

No language, follow-up or study size were imposed as restrictions in the searches. Alirocumab, evolocumab, bococizumab were all included in the analyses.

Inclusion criteria was set based on the PICOS schema. The PICOS items were as follows: (P) patients with hypercholesterolemia or CVD; (I) PCSK9 inhibitors, evaluated the efficacy and safety of PCSK9 inhibitors (alirocumab or evolocumab or bococizumab); (C) control, evaluated the efficacy and safety of control (placebo or usual care or ezetimibe); (S) randomized controlled trials (RCTs).

The exclusion criteria: abstracts, reviews, and case reports; no report of efficacy and safety assessments.

\section{Data extraction}

The following data were extracted: sample size, age, design, follow-up duration, lipid profiles (LDL-C, total cholesterol [TC], triglycerides [TG], high-density lipoprotein cholesterol [HDL-C]), unstable angina (UA), myocardial infarction (MI), stroke, injection site reaction, myalgia.

\section{Statistical analysis}

Review Manager software 5.3 was used to calculate all statistical analyses. $\mathrm{I}^{2}$ statistic were used to assess heterogeneity in the analysis. If $\mathrm{I}^{2}<50 \%$, a fixed-effect model was used; otherwise, a random-effect model was applied. Publication bias was examined by using the funnel plot. For dichotomous data, odds ratios were used. Continuous data (lipid outcomes) were expressed as mean difference of percentage change from baseline and $95 \%$ confidence interval $(\mathrm{CI}) . \mathrm{P}<0.05$ was considered statistically significant. The risk of bias was estimated by using the parameters: sequence generation, concealment of group allocation, blinding during outcome assessment, selective reporting and intention-to-treat analysis [6].

\section{Results}

Initially, a total of 1820 studies were searched, of which 82 studies were excluded because of duplication and 893 studies failed to meet the inclusion criteria. Finally, a total of 45 RCTs involving 97,297 patients were included. Of these, 21 RCTs were treated with alirocumab, $21 \mathrm{RCT}$ s were treated with evolocumab, and 3 RCTs were treated with bococizumab. The study selection flow diagram is shown in Figure 1. Characteristics of the included studies are shown in Supplementary Table S1 ([S1-S45]).

\section{Lipid profile}

Triglycerides, TC, LDL-C, and HDL-C were reported in 42 studies with a total of 92,681 patients, of which 21 studies were treated with alirocumab, 18 studies were treated with evolocumab and 3 studies were treated with bococizumab. Compared with control group, alirocumab reduced LDL-C by $-51.29 \%$ (95\% CI -55.83 to $-46.75, \mathrm{p}<0.05)$, TC by $-30.31 \%$ (95\% CI -34.26 to $-26.36, \mathrm{p}<0.05)$, TG by $-10.31 \%$ (95\% CI -13.81 to $-6.81, \mathrm{p}<0.05)$ and increased HDL-C by $5.63 \%$ (95\% CI 4.86 to $6.40, \mathrm{p}<0.05)$. Compared with control group, evolocumab reduced LDL-C by $-53.99 \%$ (95\% CI -58.45 to $-49.54, \mathrm{p}<0.05)$, TC by $-34.2 \%$ (95\% CI -36.18 to $-32.21, \mathrm{p}<0.05)$, TG by $-8.86 \%$ (95\% CI -13.17 to $-4.55, \mathrm{p}<0.05)$ and increased HDL-C by $7.05 \%$ (95\% CI 5.55 to 8.54, p < 0.05). Compared with control group, bococizumab reduced LDL-C by $-56.96 \%$ (95\% CI -60.69 to $-53.23, \mathrm{p}<0.05)$, TC by $-38.96 \%$ (95\% CI -43.33 to $-34.58, \mathrm{p}<0.05)$, TG by $-17.64 \%$ (95\% CI -20.79 to -14.48 , p < 0.05 ) and increased HDL-C by $5.98 \%$ (95\% CI 4.86 to $7.11, \mathrm{p}<0.05)$ (Table 1). 


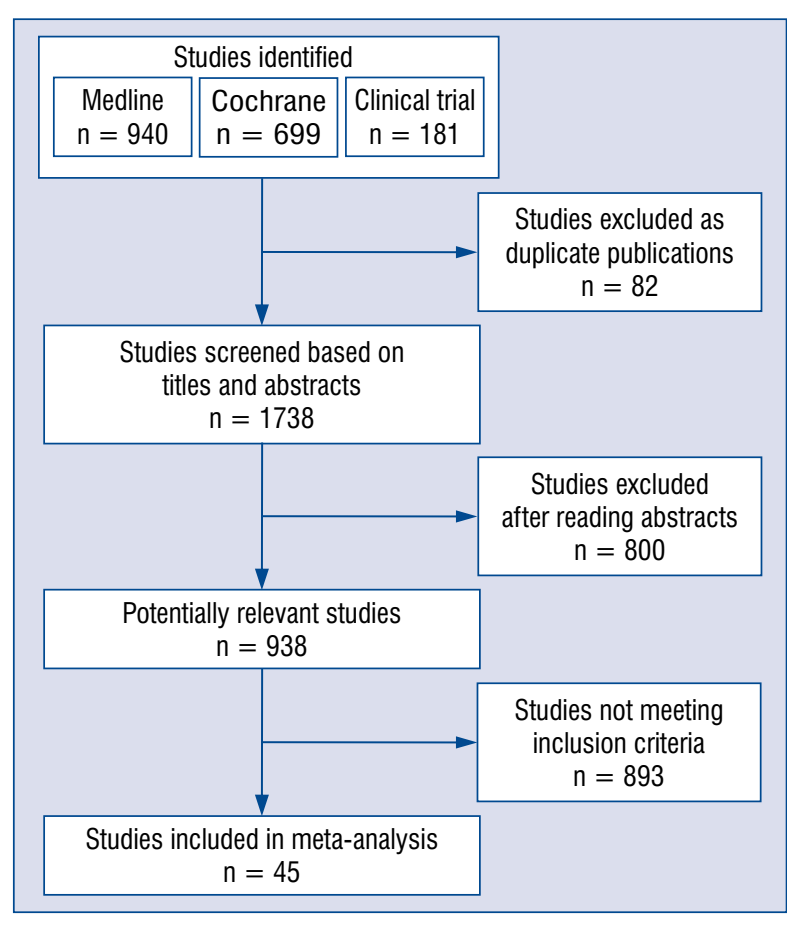

Figure 1. Selection flow diagram. In total, 1820 studies were identified. Finally, 45 studies were selected.

\section{Cardiac disorders}

Unstable angina and MI were considered as cardiac disorders. UA were reported in 13 studies with a total of 57,717 patients, of which 6 studies treated with alirocumab, 4 studies treated with evolocumab and 3 studies treated with bococizumab. MI were reported in 16 studies with a total of 90,355 patients, of which 8 studies treated with alirocumab, 6 studies treated with evolocumab and 2 studies treated with bococizumab. UA was less common in the alirocumab group (odds ratio [OR] $0.69,95 \%$ CI 0.48 to $0.98, \mathrm{p}<0.05$ ) (Fig. $2 \mathrm{~A}$ ), as was the frequency of MI (OR $0.85,95 \%$ CI 0.76 to 0.95, $\mathrm{p}<0.05$ ) (Fig. 2B). There was no significant difference in the risk of UA between evolocumab and control group (OR 0.66, 95\% CI 0.42 to 1.03 , $\mathrm{p}>0.05$ ) (Fig. 2C). However, evolocumab was associated with lower risk of MI (OR $0.73,95 \%$ CI 0.65 to $0.82, \mathrm{p}<0.05$ ) (Fig. 2D). No statistically significant difference in UA (OR $0.82,95 \% \mathrm{CI}$ 0.67 to $1.00, \mathrm{p}=0.05$ ) (Fig. $2 \mathrm{E}$ ) and MI (OR 0.94, $95 \%$ CI 0.78 to $1.14, \mathrm{p}>0.05$ ) (Fig. $2 \mathrm{~F}$ ) was found between bococizumab and control.

\section{Stroke}

The incidence of stroke was significantly lower in alirocumab (OR 0.76, 95\% CI 0.60 to 0.97 , $\mathrm{p}<0.05$ ) (Fig. 3A), evolocumab (OR 0.79, 95\% CI

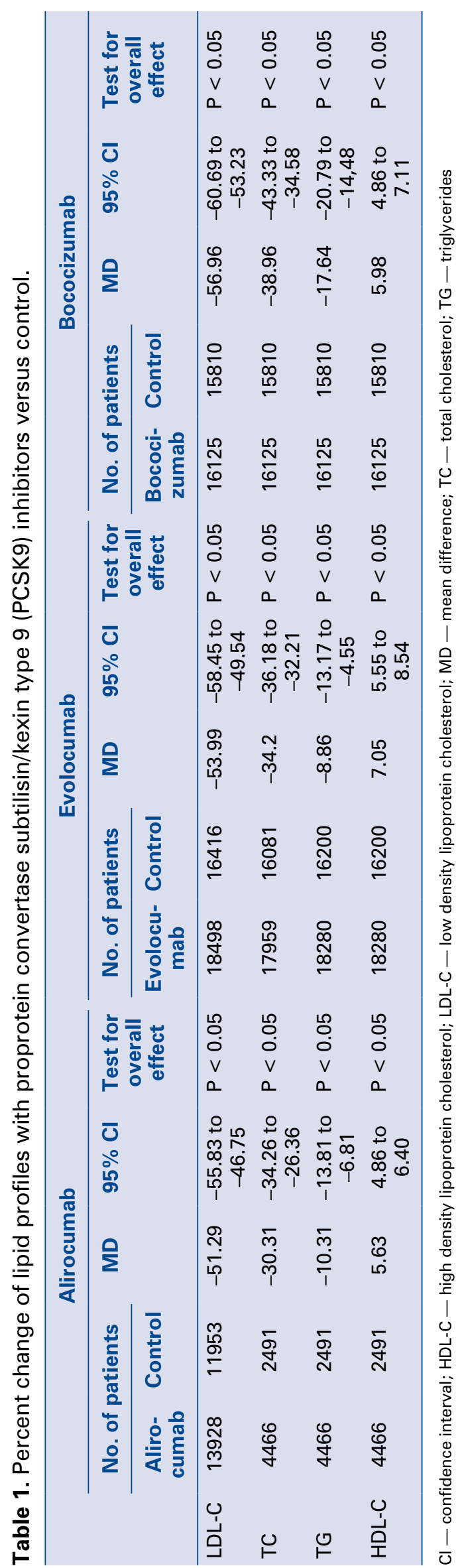




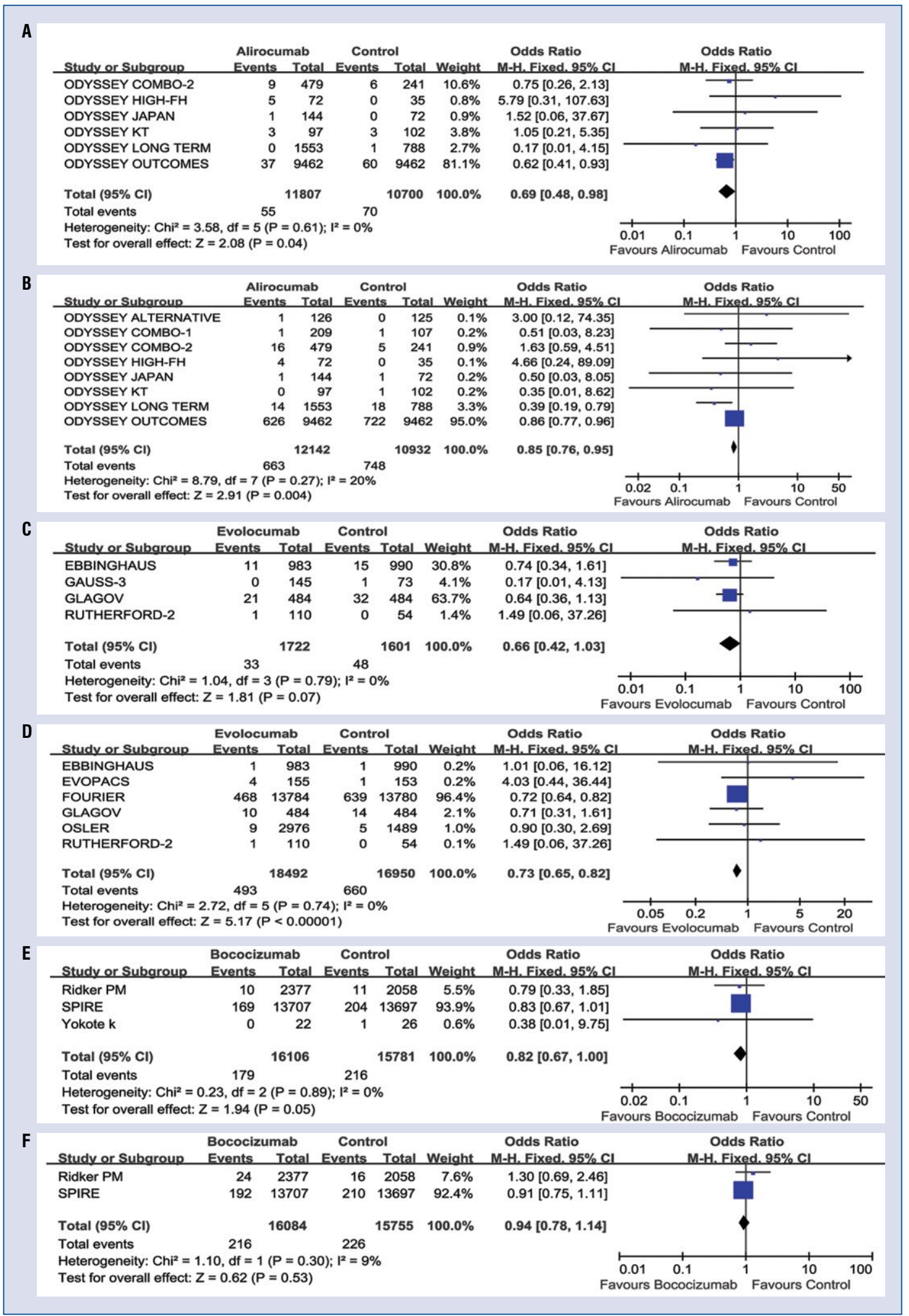

Figure 2. Forest plots of cardiac disorders with proprotein convertase subtilisin/kexin type 9 (PCSK9) inhibitors versus control. The odds ratio (OR) of unstable angina (UA) in alirocumab and control group differ significantly (OR $0.69,95 \%$ confidence interval $[\mathrm{Cl}] 0.48$ to $0.98, \mathrm{p}<0.05)(\mathrm{A})$. The OR of myocardial infarction (MI) in alirocumab and control group differ significantly (OR $0.85,95 \% \mathrm{Cl} 0.76$ to $0.95, \mathrm{p}<0.05)$ (B). There was no significant difference in the risk of UA between evolocumab and control group (OR $0.66,95 \% \mathrm{Cl} 0.42$ to $1.03, \mathrm{p}>0.05$ ) (C). Evolocumab was associated with lower risk of $\mathrm{MI}(\mathrm{OR} 0.73,95 \% \mathrm{Cl} 0.65$ to $0.82, \mathrm{p}<0.05)$ (D). No statistically significant difference in UA (OR $0.82,95 \% \mathrm{Cl} 0.67$ to $1.00, \mathrm{p}=0.05)(\mathrm{E})$ and $\mathrm{MI}$ (OR $0.94,95 \% \mathrm{Cl} 0.78$ to $1.14, \mathrm{p}>0.05)$ (F) was found between bococizumab and control. 


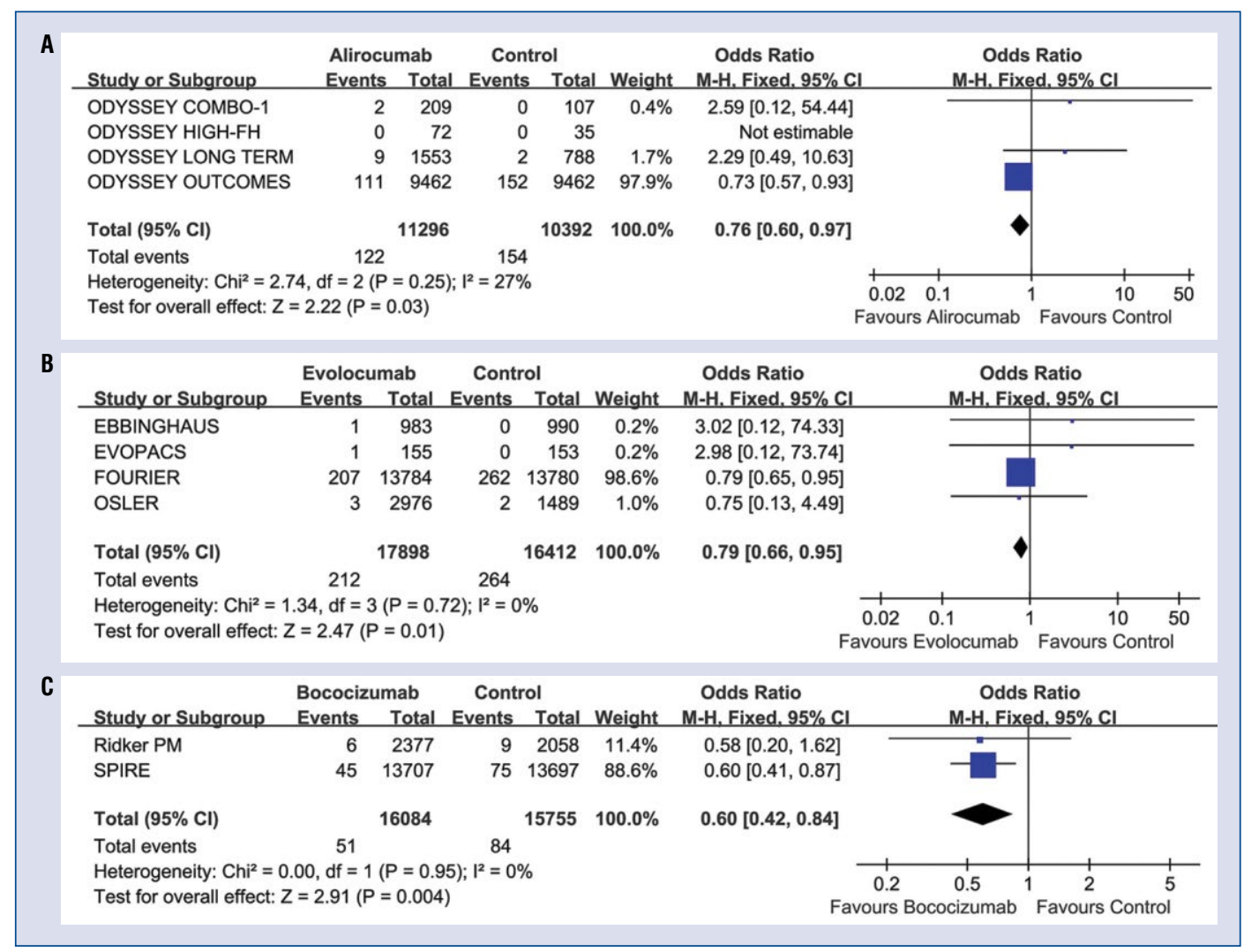

Figure 3. Forest plots of stroke with proprotein convertase subtilisin/kexin type 9 (PCSK9) inhibitors versus control. Stroke were reported in 10 studies with a total of 87,837 patients, of which 4 studies treated with alirocumab, 4 studies treated with evolocumab and 2 studies treated with bococizumab. The incidence of stroke was significantly lower in alirocumab (odds ratio [OR] 0.76, 95\% confidence interval $[\mathrm{Cl}] 0.60$ to $0.97, \mathrm{p}<0.05$ ) (A), evolocumab (OR $0.79,95 \%$ $\mathrm{Cl} 0.66$ to $0.95, \mathrm{p}<0.05)$ (B) and bococizumab (OR 0.60, 95\% $\mathrm{Cl} 0.42$ to $0.84, \mathrm{p}<0.05)$ (C) group than in control group.

0.66 to $0.95, \mathrm{p}<0.05$ ) (Fig. 3B), and bococizumab (OR $0.60,95 \%$ CI 0.42 to $0.84, \mathrm{p}<0.05$ ) (Fig. $3 \mathrm{C}$ ) group than in control group.

\section{Safety}

The safety concerns included injection-site reactions and myalgia. Injection-site reactions included dryness, discoloration, erythema, exfoliation, hematoma, hemorrhage, edema, pain, rash, sweeling, urticaria, vesicles or bruising at the injection site. The incidence of injection-site reactions was significantly higher in alirocumab (OR 1.68, 95\% CI 1.45 to $1.93, \mathrm{p}<0.05$ ) (Fig. 4A), evolocumab (OR 1.64, 95\% CI 1.41 to $1.91, \mathrm{p}<0.05$ ) (Fig. 4B), and bococizumab (OR 8.03, 95\% CI 6.85 to 9.41 , $\mathrm{p}<0.05$ ) (Fig. 4C) group than in control group. Compared with control group, alirocumab (OR $1.18,95 \%$ CI 0.92 to 1.53 , p > 0.05) (Fig. 5A), evolocumab (OR $1.09,95 \%$ CI 0.85 to $1.38, \mathrm{p}>0.05$ ) (Fig. 5B), and bococizumab (OR 1.05, 95\% CI 0.92 to $1.20, \mathrm{p}>0.05$ ) (Fig. $5 \mathrm{C}$ ) group had no significant difference in the incidence of myalgia.

\section{Discussion}

In the current study, it was found that PCSK9 inhibitors could lead to marked reduction in LDL-C, $\mathrm{TC}, \mathrm{TG}$, and increase in HDL-C. Alirocumab, evolocumab and bococizumab could reduce LDL-C $>50 \%$, increase HDL-C > 5\%. PCSK9 inhibitors could ameliorate the lipid profile. The present results about lipid changes were consistent with the meta-analysis by Zhang et al. [7].

This meta-analysis has shown that alirocumab and evolocumab probably have beneficial effects on cardiovascular outcomes. The current study demonstrated that evolocumab could reduce the incidence of MI, but did not have significant benefit with respect to UA. It was considered that this result should be interpreted with caution.

According to available research, this is the first meta-analysis to demonstrate the efficacy and safety of bococizumab. Injection-site reactions occurred in $8.4 \%$ of patients with bococizumab. Meanwhile, the rate of injection-site reactions for 


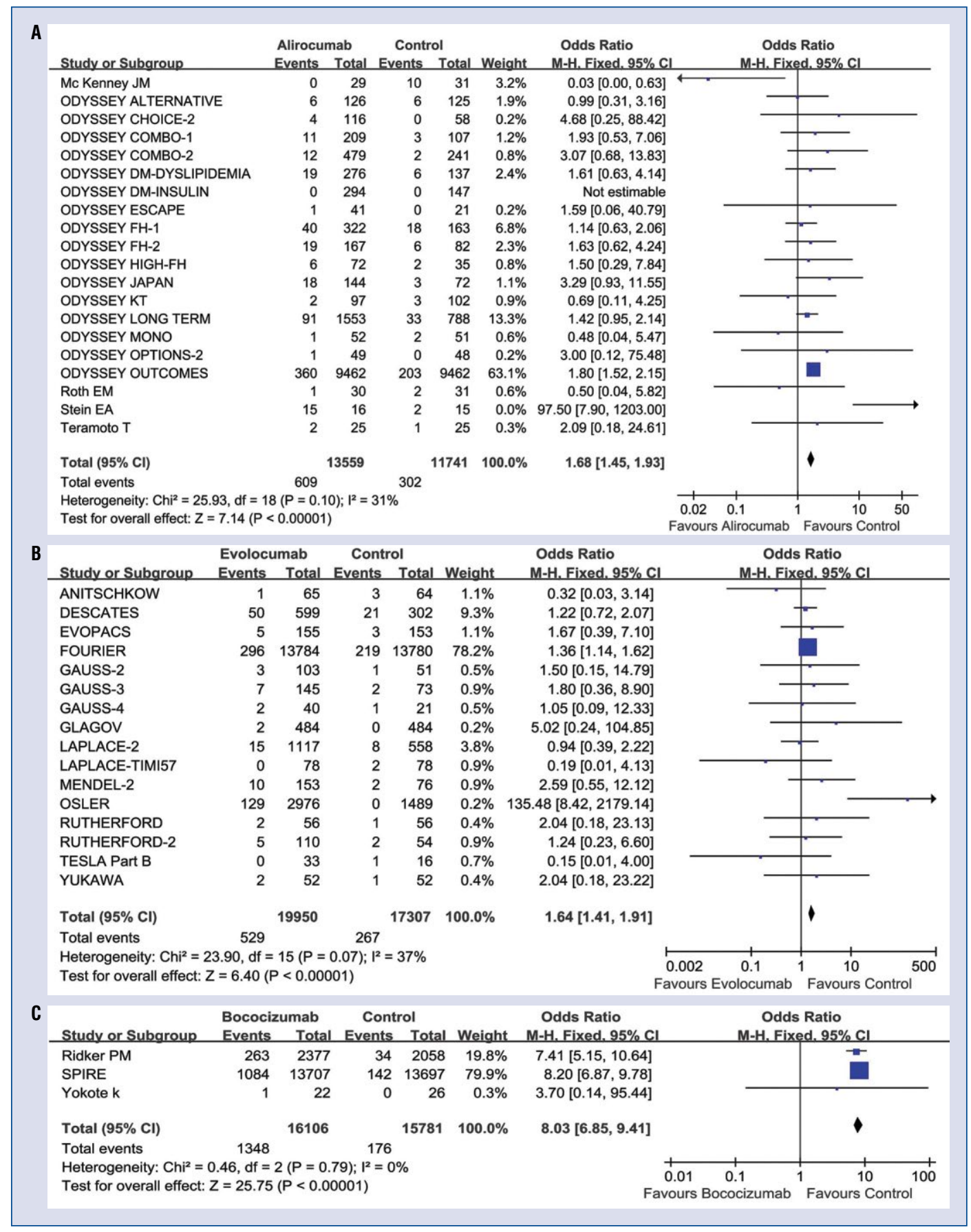

Figure 4. Forest plots of injection site reaction with proprotein convertase subtilisin/kexin type 9 (PCSK9) inhibitors versus control. Injection-site reactions were reported in 38 studies with a total of 94,444 patients, of which 19 studies treated with alirocumab, 16 studies treated with evolocumab and 3 studies treated with bococizumab. The incidence of injection-site reactions in alirocumab (odds ratio $[\mathrm{OR}] 1.68,95 \%$ confidence interval $[\mathrm{Cl}] 1.45$ to $1.93, \mathrm{p}<0.05)(\mathrm{A})$, evolocumab (OR 1.64, 95\% Cl 1.41 to $1.91, \mathrm{p}<0.05$ ) (B) and bococizumab (OR 8.03, 95\% $\mathrm{Cl} 6.85$ to 9.41, p < 0.05$)(\mathbf{C})$ group was significantly higher than in control group.

alirocumab and evolocumab therapy were $4.5 \%$ and $2.7 \%$, respectively. Alirocumab, evolocumab and bococizumab had no significant difference in the incidence of myalgia. In the current analysis, it was found that bococizumab was not associated with reduction of cardiovascular events. These findings observed in the current analysis was similar to that observed in the SPIRE study [2]. It was thought that this was the reason why the sponsors decided to discontinue the clinical development of 


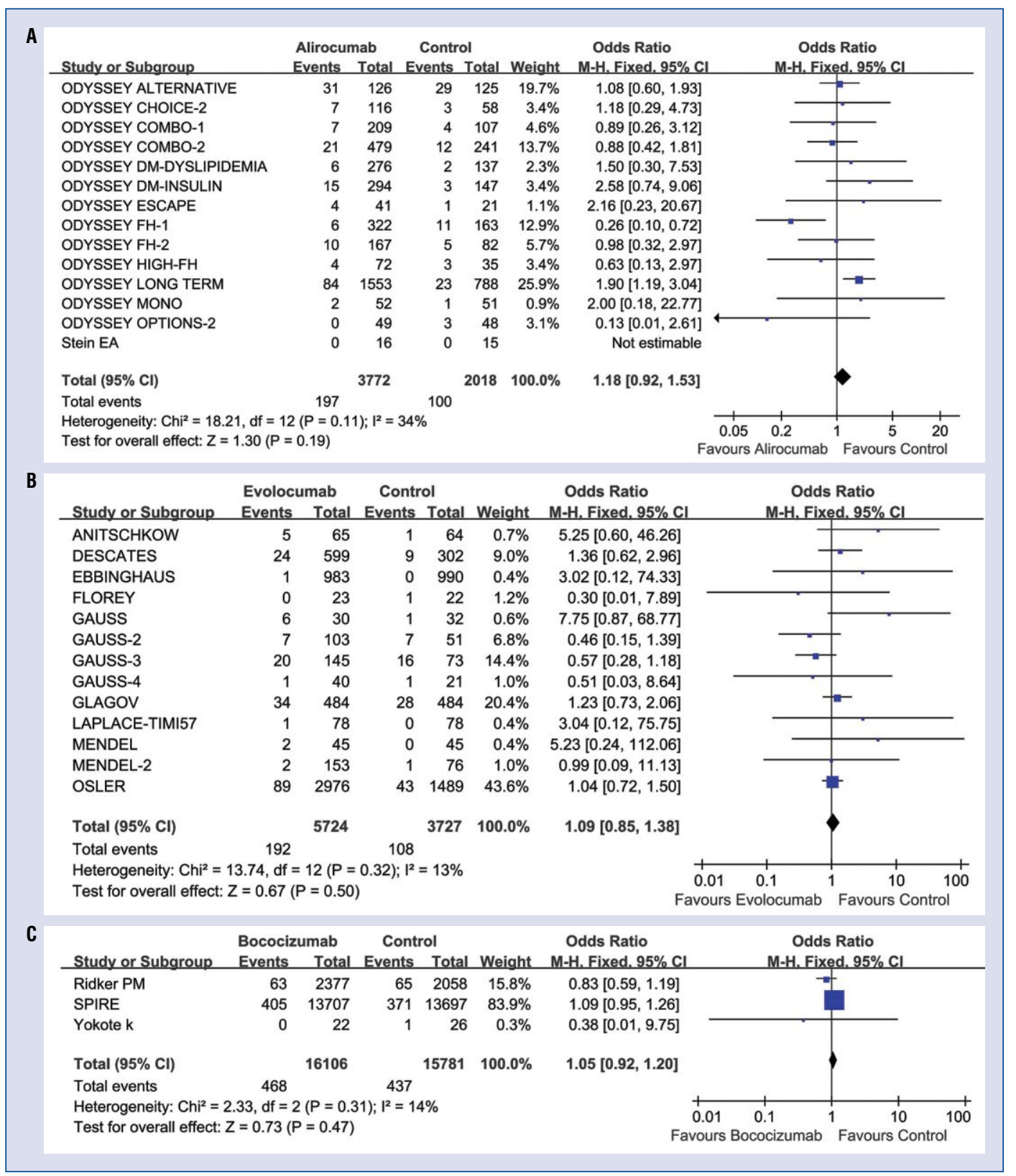

Figure 5. Forest plots of myalgia with proprotein convertase subtilisin/kexin type 9 (PCSK9) inhibitors versus control. Myalgia was reported in 30 studies with a total of 47,128 patients, of which 14 studies treated with alirocumab, 13 studies treated with evolocumab and 3 studies treated with bococizumab. Compared with control group, alirocumab group (odds ratio [OR] 1.18, 95\% confidence interval $[\mathrm{Cl}] 0.92$ to $1.53, \mathrm{p}>0.05$ ) (A), evolocumab group (OR $1.09,95 \% \mathrm{Cl} 0.85$ to $1.38, \mathrm{p}>0.05$ ) (B) and bococizumab group (OR 1.05, 95\% Cl 0.92 to $1.20, \mathrm{p}>0.05)(\mathrm{C})$ had no significant difference in the incidence of myalgia.

bococizumab. More RCTs are needed to provide more evidence to prove the efficacy and safety of bococizumab.

PCSK9 is expressed in atherosclerotic plaques and might promote atherosclerosis by stimulating inflammation and endothelial dysfunction [8]. The present study found that alirocumab and evolocumab could reduce the risk of cardiovascular events and stroke, which may be related to their ability to ameliorate the blood lipid profile, inhibit PCSK9 expression in plaques, and inhibit inflammation. These findings were very encouraging and demonstrated conclusive evidence in favor of alirocumab and evolocumab therapy for CVD patients with acceptable safety concerns. In realworld practice, evolocumab has been prescribed 
with favorable safety and tolerability outcomes [9]. However, more randomized clinical evidence was needed to explore the efficacy of bococizumab.

\section{Limitations of the study}

There are several limitations that should be taken into account in this analysis. First, the dose of PCSK9 inhibitors and different follow-up duration may have affected heterogeneity to the results. The shortest follow-up period was 8 weeks, the longest was 134 weeks. Second, the pooling of data in control group was a mixture of placebo or ezetimibe. Third, definitions of efficacy and safety were nonuniform in the included studies.

\section{Clinical perspective}

In our opinion, there is enough evidence with alirocumab and evolocumab on cardiovascular events and lipid profile to approve of using them.

Adverse events of PCSK9 inhibitors were mild and acceptable in patients with CVD. PCSK9 inhibitors were generally safe and well tolerated.

\section{Conclusions}

In conclusion, the present meta-analysis revealed that, compared with no PCSK9 inhibitors management, treatment with alirocumab and evolocumab could ameliorate lipid profile in ASCVD and reduce the risk of cardiac disorders and stroke. However, injection-site reactions should be paid attention to.

\section{Acknowledgments}

All authors had access to the data and participated in writing this manuscript. This work was supported by the Qingdao Outstanding Health Professional Development Fund.

\section{Funding}

This work was supported by Qingdao Outstanding Health Professional Development Fund, Science and Technology Program for Benefiting the People in Qingdao (21-1-4-rkjk-9-nsh).

\section{Conflict of interest: None declared}

\section{References}

1. Turgeon RD, Tsuyuki RT, Gyenes GT, et al. Cardiovascular Efficacy and Safety of PCSK9 Inhibitors: Systematic Review and Meta-analysis Including the ODYSSEY OUTCOMES Trial. Can J Cardiol. 2018; 34(12): 1600-1605, doi: 10.1016/j. cjca.2018.04.002, indexed in Pubmed: 30527147.

2. Ridker PM, Revkin J, Amarenco P, et al. Cardiovascular efficacy and safety of bococizumab in high-risk patients. N Engl J Med. 2017; 376(16): 1527-1539, doi: 10.1056/NEJMoa1701488, indexed in Pubmed: 28304242.

3. Ridker PM, Tardif JC, Amarenco P, et al. Lipid-Reduction variability and antidrug-antibody formation with bococizumab. N Engl J Med. 2017; 376(16): 1517-1526, doi: 10.1056/NEJMoa1614062, indexed in Pubmed: 28304227.

4. Moher D, Liberati A, Tetzlaff J, et al. Preferred reporting items for systematic reviews and meta-analyses: the PRISMA statement. PLoS Medicine. 2009; 6(7): e1000097, doi: 10.1371/journal.pmed.1000097.

5. Higgins JPT, Altman DG, Gøtzsche PC, et al. The Cochrane Collaboration's tool for assessing risk of bias in randomised trials. BMJ.2011; 343: d5928, doi: 10.1136/bmj.d5928, indexed in Pubmed: 22008217.

6. Higgins JPT, Green S (eds). Cochrane Handbook for Systematic Reviews of Interventions, Version 5.1.0 [updated March 2011]. The Cochrane Collaboration. 2011. http://www.cochrane-handbook.org (accessed 21 March 2011).

7. Zhang XL, Zhu QQ, Zhu Li, et al. Safety and efficacy of antiPCSK9 antibodies: a meta-analysis of 25 randomized, controlled trials. BMC Med. 2015; 13: 123, doi: 10.1186/s12916-015-0358-8, indexed in Pubmed: 26099511.

8. Li J, Liang X, Wang Y, et al. Investigation of highly expressed PCSK9 in atherosclerotic plaques and ox-LDL-induced endothelial cell apoptosis. Mol Med Rep. 2017; 16(2): 1817-1825, doi: 10.3892/mmr.2017.6803, indexed in Pubmed: 28656218.

9. Koskinas KC, Windecker S, Pedrazzini G, et al. Design of the randomized, placebo-controlled evolocumab for early reduction of LDL-cholesterol levels in patients with acute coronary syndromes (EVOPACS) trial. Clin Cardiol. 2018; 41(12): 1513-1520, doi: 10.1002/clc.23112, indexed in Pubmed: 30421481. 